\title{
Evolution of Self-Focusing of Intense Electromagnetic Waves in Plasma
}

\author{
W. B. Mori, C. Joshi, and J. M. Dawson \\ University of California at Los Angeles, Los Angeles, California 90024 \\ and \\ D. W. Forslund and J. M. Kindel ${ }^{(a)}$ \\ Los Alamos National Laboratory, Los Alamos, New Mexico 87545 \\ (Received 17 April 1987)
}

\begin{abstract}
The self-focusing of intense electromagnetic waves in a very underdense plasma is studied in computer simulations. Relativistic self-focusing occurs initially. This is followed by "ponderomotive blowout" and filamentation at the edge of the channel walls. The self-focusing is more intense for resonant doublefrequency than for single-frequency illumination.

PACS numbers: $52.65 .+\mathrm{z}, 52.25 .-\mathrm{b}, 52.60 .+\mathrm{h}$
\end{abstract}

The self-focusing of electromagnetic waves in nonlinear media is a topic of fundamental interest. Selffocusing arises when the dielectric function (index of refraction) of a medium is lower where the intensity of the radiation is most intense. In a plasma there are three types of direct self-focusing mechanisms. These are relativistic, ${ }^{1}$ ponderomotive, ${ }^{2}$ and thermal self-focusing. ${ }^{3}$ Ponderomotive and thermal self-focusing occur when density perturbations are ultimately caused by radiation or thermal pressure, respectively, while relativistic selffocusing is driven by the increase in the electrons' mass from their quiver motion. Relativistic self-focusing, therefore, does not involve any transient behavior and, as a result, once the power threshold ${ }^{1} P_{1}=15\left(\omega_{1} / \omega_{p}\right)^{2} \mathrm{GW}$ ( $\omega_{1}$ and $\omega_{p}$ are the incident-wave and plasma frequency, respectively) is exceeded, it immediately occurs. This fact allows the incident beam's envelope to be analytically predicted for all times until the ions move. On the other hand (for ponderomotive or thermal self-focusing), only steady-state envelope solutions are analytically obtainable and these are calculated ${ }^{2}$ by use of only the weak nonlinearity in slab geometry or use of the Gaussian-profile Ansatz for cylindrical geometry. It is therefore necessary to resort to numerical analysis ${ }^{4}$ in order to study both how the beam profile approaches steady state (if ever) and how closely the beam's steady-state profile resembles the weak-nonlinearity predictions. In this Letter we use two-dimensional particle-in-cell periodic simulations to address some of these issues in the combined relativistic and ponderomotive self-focusing situation for both single- and double-frequency illumination. ${ }^{5}$

The primary results of this Letter are the following: (1) For two-frequency illuminations, the resonantly excited plasma wave enhances the self-focusing of each incident wave through its own relativistic quiver motion of the electrons. The nonlinear response of the plasma is no longer instantaneous and later light rays are influenced by preceding ones since the plasma wave's group velocity is practically zero. (2) The space-time formation of the plasma density channel is strongly influenced by the oc- currence of both relativistic and ponderomotive selffocusing, and it involves kinetic effects. (3) We find no evidence for the prediction by Sun et al., ${ }^{4}$ of complete expulsion (cavitation) of electrons from the beam region for the simulated beam widths.

The 2D electromagnetic particle-in-cell code WAVE is used for the simulations. Common parameters are $v_{1,2} / c=0.56, T_{e, i}=2.5 \mathrm{keV}$, and $L_{x, y}=60 c / \omega_{p}$, where $v_{1,2}$ are the quiver velocities of an electron and $\omega_{1,2}$ are the frequencies for each input wave. Each input wave enters with a $\cos ^{2}\left(\pi y / L_{0}\right)$ profile and the value of $L_{0}$ is varied. We begin with two simulations in which $L_{0}$ $=20 c / \omega_{p}, \tau=300 \omega_{p}^{-1}$, where $\tau$ is the incident wave's rise time, and $m_{i} / m_{e}$ is either $\infty$ or 1836. In these simulations the incident power is above the relativistic selffocusing threshold by roughly an order of magnitude.

Relativistic self-focusing is illustrated in Fig. 1(a) where the contour plot of the incident field $E_{z}$ is shown at $\omega_{p} t=330$ for fixed ions. The intensity on axis is 3 times larger on the right than the left. By this time the incident beam has already collapsed down to a width of $\approx 5 c / \omega_{p}$ in a distance of $30 c / \omega_{p}$. These compare favorably to the predictions of Sprangle, Tang, and Esarey ${ }^{1}$ using cylindrical geometry for an incident power an order of magnitude above the threshold power. Their ana-

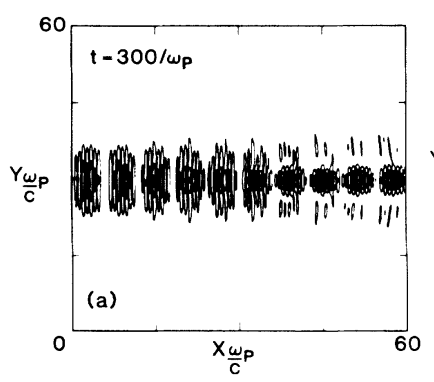

(a)

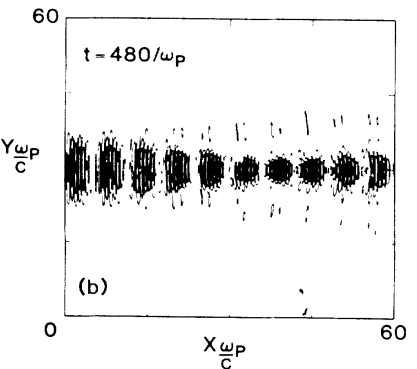

(b)
FIG. 1. The contour plot of the laser's field $E_{z}$ at (a) $\omega_{p} t=330$ for fixed ions and (b) $\omega_{p} t=480$ for mobile ions. The value of $L_{0}$ is $20 c / \omega_{p}$. 
lytic work also predicts that the waves' radius will execute oscillatory motion so that the beam will begin to expand again toward the right in Fig. 1(a). However, this behavior is not observed in the $x-y$ Cartesian-geometry simulations; instead the beam width remains relatively constant over the second half of the box. This is qualitatively similar to numerical work with the nonlinear Schrödinger equation in the same paper, where it was found that the envelope never approached its initial radius after the first collapse. This can be partly attributed to the fact that not all of the incident wave's energy self-focuses as seen by the diffraction of the outer edge of the beam in Fig. 1. It has been observed by others ${ }^{4}$ that in cylindrical geometry with saturable nonlinearities the beam breaks up into rings with roughly only the threshold power being contained in the central ring.

The self-focusing is more intense at $\omega_{p} t=330$ than at the end of the simulation, $\omega_{p} t=540$. The reason is the presence of the large-amplitude plasma wave for times between $\omega_{p} t=150$ and $\omega_{p} t=360$. The dielectric function for a linearly polarized wave in the presence of another light wave and a resonantly excited plasma wave is (cascaded modes are neglected)

$$
\epsilon=1-\frac{\omega_{p}^{2}}{\omega_{1}^{2}}\left[1+\frac{\delta n}{n}-\frac{1}{2}\left(\frac{3}{4}-\frac{\omega_{1}^{2}-\omega_{p}^{2}}{4 \omega_{1}^{2}-\omega_{p}^{2}}\right) \frac{v_{1}^{2}}{c^{2}}-\frac{1}{4}\left(3-\frac{\left(k_{1}+k_{2}\right)^{2} c^{2}}{\left(\omega_{1}+\omega_{2}\right)^{2}-\omega_{p}^{2}}\right) \frac{v_{2}^{2}}{c^{2}}-\frac{1}{4} \frac{v_{p}^{2}}{c^{2}}\right],
$$

where $\delta n / n$ is any zero-frequency electron-density perturbation, and $v_{p}$ is the quiver velocity of an electron in the plasma wave. In this simulation the ratio of the longitudinal to electromagnetic nonlinearities in Eq. (1) can be as high as $\frac{4}{3}$. This demonstrates the possible importance of the plasma wave.

We note that when the electrons respond adiabatically to the ponderomotive force, the resulting nonlinearity $\delta n / n \propto \nabla^{2}\left(v_{1}^{2} / c^{2}\right)$ is typically important only for scalelengths on the order of $c / \omega_{p}$. This nonlinearity by itself leads to an intensity threshold of $v_{1}^{2} / c^{2} \gtrsim 4$ rather than a power threshold for self-focusing. Sun et al. ${ }^{4}$ have shown that the inclusion of both types of nonlinearities can lead to final states for which there is complete expulsion of the electrons from the beam region for power levels only $10 \%$ above the standard self-focusing threshold. In the simulations there was only a slight amount of electron expulsion rather than complete evacuation.

With mobile ions the self-focusing behavior is substantially different. Later in time a channel void of both electron and ions forms, which initially enhances and eventually disrupts the self-focusing. Even though the dielectric function is not a function of the ion density, the ions are important since their mobility permits an electron-density depression to form. The effect of mobile ions is most vivid for high-power narrow beams as illustrated in Fig. $1(\mathrm{~b})$ where the $E_{z}$ contour plot is shown for $\omega_{p}(t)=480$, and in Fig. 2 where the $x-y, v_{y}$ vs $x$, and $v_{y}$ vs $y$ ion phase-space plots are given. In Fig. 1(b) the beams are seen to self-focus similarly as in Fig. 1(a), but then expand out again. The subsequent expansion occurs because the plasma is almost completely expelled as seen in Fig. 2(a), leading to little variation of $\epsilon$ across the channel except at the channel's walls.

The formation of the channel arises as follows. The ponderomotive force, by expelling electrons, creates a space-charge force which pulls out a fraction of the ions. These ions cancel part of the space charge allowing the ponderomotive force to expel more electrons, and so on. The analytic steady-state solutions assume that the plasma pressure eventually balances the ponderomotive pres- sure. In these high-intensity simulations where $v_{0 i} / v_{\text {th }}$ $\gg 1$ the ions acquire velocities well in excess of the ion acoustic velocity $c_{s}=\left[\left(K T_{e}+3 K T_{i}\right) / M\right]^{1 / 2}$ as seen in Fig. 2(b). In the simulations the peak ion velocities are $\simeq 5-10$ times $c_{s}$. The electrons do heat up in the interaction region from the Raman instability and this increases the relative importance of the thermal pressure. The ions and electrons then move back through the background plasma forming an ever steepening ion wave front and this is shown in Fig. 2(c). In some situations it should be possible to excite a high-Mach-number electrostatic shock. ${ }^{6}$ Eventually a channel forms void of plasma with discontinuous boundaries. When this occurs $\epsilon$ does not vary across the channel and so the beam ceases to self-focus. The density jumps from zero to $2-3$ times the background at the wall in a few $c / \omega_{p}$. So for a plasma which is initially $5 \%$ critical, no critical or quarter-critical surface is present so neither resonance absorption ${ }^{7}$ nor the $2 \omega_{p}$ instability $^{8}$ can occur. The wave front eventually steepens and exhibits $x$-type wave-breaking behavior. ${ }^{9}$ In other simulations, where the ponderomotive force is not as large, the front moves out but does not break as soon. When the wave front breaks, the ion transverse temperature increases, and the density gradient at the channel's edge relaxes.
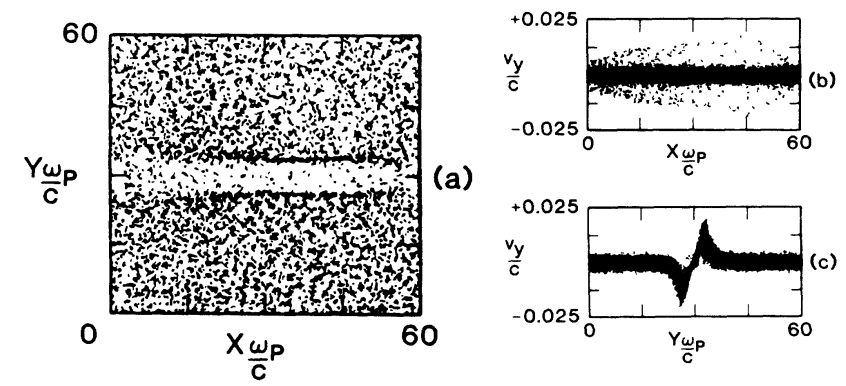

FIG. 2. The (a) $x-y$, (b) $v_{y}$ vs $x$, and (c) $v_{y}$ vs $y$ ion phasespace plots at $\omega_{p} t=420$. Simulation parameters are $v_{1} / c$ $=v_{2} / c=0.56, \tau=300 \omega_{p}^{-1}$, and $L_{0}=20 c / \omega_{p}$. 
The time it takes for the channel to form can be crudely estimated by the calculation of how long it takes an ion to move a fraction of the beam's width, under the influence of the ponderomotive force. Following this procedure we find

$$
\omega_{p i} t=\frac{2^{1 / 2} \alpha^{1 / 2} L_{0}}{\left\langle\left|v_{01} / c\right|^{2}+\left|v_{02} / c\right|^{2}+\left|v_{p} / c\right|^{2}\right\rangle^{1 / 2}},
$$

where $L_{0}$ is the beam's width and $\alpha$ is the fraction of the beam's width that an ion moves. Use of $L_{0}=20, \alpha=\frac{1}{4}$, and $\left\langle\Sigma\left(v_{j} / c\right)^{2}\right\rangle=1$ gives an approximate time of $\omega_{p i} t$ $=14$ and a corresponding ion velocity of $v_{i} / c=0.013$. In the simulations the values were $\cong 10$ and $\cong 0.025$. The discrepancy probably arises because $L_{0}$ gets smaller as the beam self-focuses. This effect can be seen in Fig. 2(b) where in the $v_{y}$ vs $x$ phase-space plot the velocities are largest on the right.

A simulation was done where the spot size was doubled to $L_{0}=40 c / \omega_{p}$ so that the laser's power was increased while the peak intensity was kept constant. The increase in power leads to greater amplification in longer distances from relativistic self-focusing. At $\omega_{p} t=307$, when $L_{0}=40 c / \omega_{p}$ the peak value of $e E_{z} / m c \omega_{j}$ at the right-hand boundary is 7.0 (the input value is 5.0). This is in contrast to the $L_{0}=20 \mathrm{c} / \omega_{p}$ case where $E_{z}$ is 5.5 at the same time. In terms of intensity this is roughly a factor of 2 difference. The focused widths are roughly (4-5) $c / \omega_{p}$ in both cases. The channel formation exhibits similar characteristics. However, since the initial ponderomotive force is smaller the channel takes longer to form and the wave form does not steepen as quickly. Therefore, the channel density has a gentler gradient leading to stronger whole beam self-focusing. The width at the right-hand boundary eventually approaches $2 c / \omega_{p}$ with a factor of 4 increase in intensity by $\omega_{p} t=530$ as seen in Fig. 3(a).

The larger value of $L_{0}$ leads to ponderomotive filamentation $^{2}$ late in the simulation. This is evident in Fig. 3(b) where the contour plot of $E_{z}$ leaving the right-hand boundary is given at $\omega_{p} t=770$. The amplification of the field is not as large as at either $\omega_{p} t=307$ or 530. This is because the energy is divided into two filaments rather than one. The filamentation is seeded from the localized regions of variation in $\epsilon$ at the channel boundaries. Eventually this leads to density filamentation in the inside edge of the channel.

When the laser's rise time is increased from $\tau$ $=300 \omega_{p}^{-1}$ to $\tau=800 \omega_{p}^{-1}$, the amount of amplification in intensity over the length of the box decreased from 4.5 to 3. It seems, from these simulations, that self-focusing (amplification) is strongest when the onset of relativistic and ponderomotive effects are clearly separated in time. In this longer-rise-time simulation there is not as clear a separation in time between the two types of self-focusing. Late in the simulation filamentation is also observed, just as in the $\tau=300 \omega_{p}^{-1}$ simulation. The filamentation is

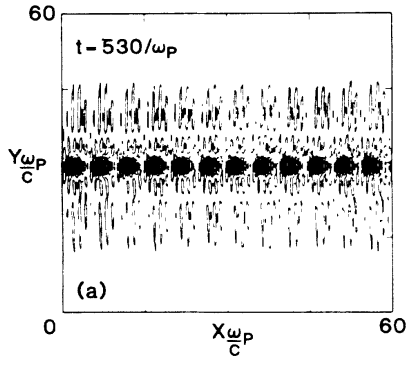

(a)

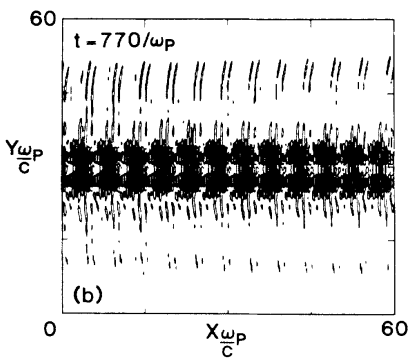

(b)
FIG. 3. The contour plot of the laser's field $E_{z}$ leaving the right-hand boundary for $L_{0}=40 c / \omega_{p}$ at (a) $\omega_{p} t=530$ and (b) $\omega_{p} t=770$.

observed around $\omega_{p} t=850$ or at the peak of the laser rise time. The amplification for filamentation is larger than in the $\tau=300 \omega_{p}^{-1}$ simulation. The reason is that in this case the channel is not as wide and the walls are not as sharply defined so that the whole beam can still be amplified.

A single-frequency $\left(\omega_{1}=\omega_{2}=5 \omega_{p}\right)$ simulation was conducted to ellucidate the differences between singleand double-frequency illuminations. Self-focusing is observed in the single-frequency case at $\omega_{p} t=500$; but it is not nearly as strong as in the double-frequency case where the onset of self-focusing is observed earlier $\left(\simeq \omega_{p} t=450\right)$. Since ponderomotive effects are not as important at this time this is additional evidence of resonant relativistic self-focusing. At $\omega_{p} t=720$ the ions have already been accelerated to 5 times the ion acoustic velocity in the double-frequency case while they are just beginning to be accelerated in the single-frequency case. This difference arises since the time-integrated ponderomotive force is larger in the double-frequency case, both because the light waves have self-focused sooner as discussed earlier and because the ponderomotive force is larger from the contribution of the plasma wave. This latter effect, resonant ponderomotive self-focusing, has been observed experimentally ${ }^{10}$ in a low-intensity regime. It should be noted that the plasma wave, being $\propto v_{1} v_{2} / c^{2}$, is more localized in space than the incident waves; and hence, it has sharper gradients. An additional qualitative difference is that the maximum ion velocity is constant along $x$ for a single frequency while it varies along $x$ for two frequencies. In both simulations filamentation eventually occurs but it occurs first in the double-frequency case by roughly an $\omega_{p i}^{-1}$.

The simulations model a certain section of the plasma with the laser pulse moving through it. On the other hand, what is of interest for the plasma beat-wave accelerator ${ }^{5}$ is the evolution of the laser pulse as it moves into fresh plasma. In order to obtain a better idea of the self-focusing behavior of the laser beams for longer propagation distances, a simulation with parameters similar to the $L_{0}=20 c / \omega_{p}$ case, except for the system size which 


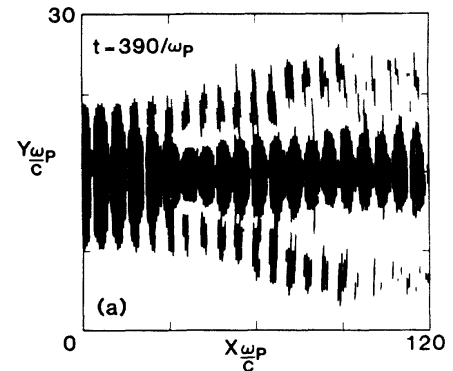

(a)

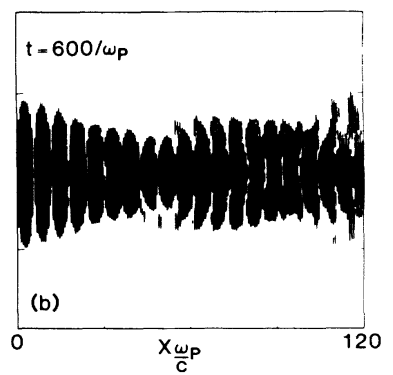

(b)
FIG. 4. The contour plot of the laser's field $B_{y}$ at (a) $\omega_{p} t=390$ and (b) $\omega_{p} t=600$ for $\tau=300 \omega_{p}^{-1}$ and $L_{0}=20 c / \omega_{p}$.

was doubled in $x$ and halved in $y$, was carried out. The results are summarized in Fig. 4 where the contour plot of the laser's magnetic field $B_{y}$ is shown at $\omega_{p} t=390$ and 600. At $\omega_{p} t=390$, the laser is at full intensity across almost the entire box since $\tau=300 \omega_{p}^{-1}$ and $L_{x}=120 c / \omega_{p}$. In Fig. 4(a) the effect of relativistic self-focusing is evident. The laser collapses down to its narrowest point at $x=30 c / \omega_{p}$ and then remains reasonably constant at a width of $\cong 5 c / \omega_{p}$ in the second half of the box. The left half of Fig. 4(a) is essentially identical to Fig. 1(b). Even though by this time a plasma channel is forming on the left, the laser profile is still determined primarily by relativistic effects. As mentioned earlier, not all of the laser energy is focused, as seen by the outer contours in Fig. 4(a).

The effect of the channel formation is seen in Fig. 4(b). The point to which the laser wave first converges has moved slightly to the right. As the channel width continues to increase this point moves even further to the right. After the initial focus the beam expands and then refocuses, as seen in Fig. 4(b). This oscillatory behavior is exhibited in the plasma channel as well. This has bec predicted by both Felber ${ }^{2}$ and $\mathrm{Max}^{2}$ for the steady-sta1 problem. Comparison of our work with those of Felbe and Max is difficult since in the simulations both th channel's shape and width are evolving even towards th end of the simulations.

Last, it should be noted that the work in this Lette1 differs from the numerical work of Jones et al., ${ }^{4}$ both in the region of parameter space studied and in the approximations used. Jones et al. examined single-frequency illumination in nearly critically dense plasmas, whereas here both single- and double-frequency illumination in very underdense plasmas are examined. Furthermore, Jones et al. neglected electron and ion kinetic effects and used time steps on the order of $\omega_{p i}$.

We gratefully acknowledge useful conversations with T. Katsouleas, R. Bingham, A. B. Langdon, and F. F. Chen. We thank C. J. McKinstrie for bringing to our attention an error in Eq. (1). This work was supported by U.S. Department of Energy Contract No. DE-AS0383-ER 40120, National Science Foundation Grants No. ECS-83-10972 and No. PHY 85-12390, and the Los Alamos National Laboratory Graduate Research Assistant program.

\footnotetext{
(a) Present address: Mission Research Corporation, 901 18th Street, Los Alamos, NM 87545.

${ }^{1}$ C. E. Max, J. Arons, and A. B. Langdon, Phys. Rev. Lett.
} 33, 209 (1974); P. Sprangle, C. M. Tang, and E. Esarey, IEEE Trans. Plasma Sci. 15, 2 (1987), Special Issue on Plasma Based High Energy Accelerators, edited by T. Katsouleas; G. Schmidt and W. Horton, Comments Plasma Phys. Controlled Fusion 9, 85 (1985).

${ }^{2}$ P. K. Kaw, G. Schmidt, and T. Wilcox, Phys. Fluids 16, 1522 (1973); C. Max, Phys. Fluids 19, 74 (1976); F. S. Felber, Phys. Fluids 23, 1410 (1980); W. L. Kruer, Comments Plasma Phys. Controlled Fusion 9, 63 (1985).

${ }^{3}$ F. W. Perkins and E. J. Valeo, Phys. Rev. Lett. 32, 1234 (1974); V. K. Tripathi and L. A. Pitale, J. Appl. Phys. 48, 3288 (1977); R. S. Craxtron and R. L. McCroy, J. Appl. Phys. 56, 108 (1984).

${ }^{4}$ D. A. Jones, E. L. Kane, P. Lalousis, P. Widles, and H. Hora, Phys. Fluids 25, 2295 (1982); G. Z. Sun, E. Ott, Y. C. Lee, and P. Guzdar, Phys. Fluids 30, 526 (1987); A. Schmitt and R. S. B. Ong, J. Appl. Phys. 54, 300 (1983); B. F. Lasinski and A. B. Langdon, Laser Program Annual Report No. UCRL-50021-79, 1979 (unpublished), pp. 3-45, and references therein; A. B. Langdon, B. F. Lasinski, and C. Max, Laser Program Annual Report No. UCRL-50021-74, 1974 (unpublished), p. 410.

${ }^{5}$ C. Joshi et al., Nature (London) 311, 525 (1984); T. Tajima and J. M. Dawson, Phys. Rev. Lett. 43, 267 (1979); D. W. Forslund et al., Phys. Rev. Lett. 54, 558 (1985); W. B. Mori, Ph.D. dissertation, University of California at Los Angeles, 1987 (unpublished).

${ }^{6}$ D. W. Forslund and C. R. Shonk, Phys. Rev. Lett. 25, 1699 (1970).

${ }^{7}$ Kevin Quest, private communication.

${ }^{8}$ A. B. Langdon and B. F. Lasinski, Phys. Rev. Lett. 34, 934 (1975).

${ }^{9}$ D. W. Forslund, J. M. Kindel, and E. L. Lindman, Phys. Rev. Lett. 29, 249 (1972).

${ }^{10}$ C. Joshi, C. E. Clayton, and F. F. Chen, Phys. Rev. Lett. 48, 874 (1982). 

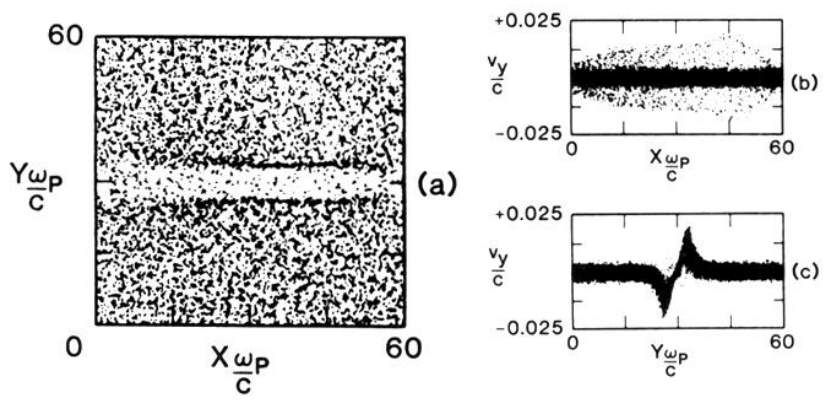

FIG. 2. The (a) $x-y$, (b) $v_{y}$ vs $x$, and (c) $v_{y}$ vs $y$ ion phasespace plots at $\omega_{p} t=420$. Simulation parameters are $v_{1} / c$ $=v_{2} / c=0.56, \tau=300 \omega_{p}^{-1}$, and $L_{0}=20 c / \omega_{p}$. 


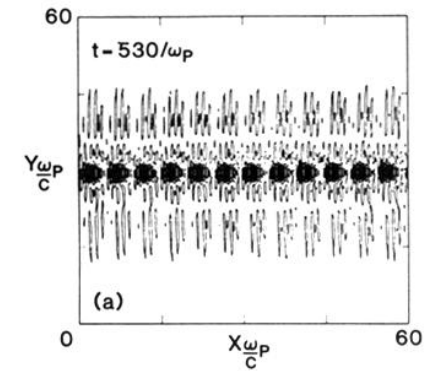

(a)

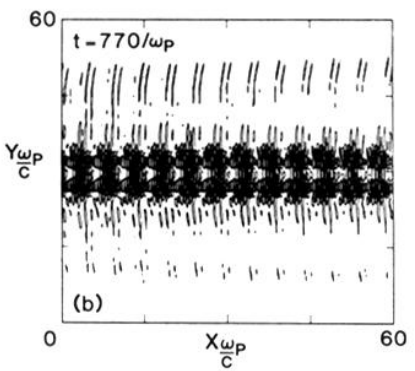

(b)

FIG. 3. The contour plot of the laser's field $E_{z}$ leaving the right-hand boundary for $L_{0}=40 c / \omega_{p}$ at (a) $\omega_{p} t=530$ and (b) $\omega_{p} t=770$. 


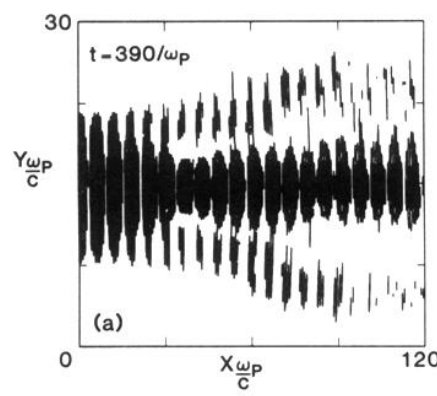

(a)

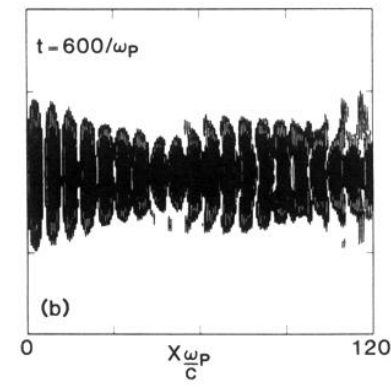

(b)

FIG. 4. The contour plot of the laser's field $B_{y}$ at (a) $\omega_{p} t=390$ and (b) $\omega_{p} t=600$ for $\tau=300 \omega_{p}^{-1}$ and $L_{0}=20 c / \omega_{p}$. 